\title{
PRECISION DIFFERENTIAL CCD PHOTOMETRY
}

\author{
L. A. Balona \\ South African Astronomical Observatory
}

\begin{abstract}
In this paper we describe the technique and reduction procedure in use at the SAAO for differential CCD photometry. Much of this work involves the detection of microvariables in galactic and Magellanic Cloud open clusters. The large number of frames requires automated cleaning and reduction software (principally the DoPhot package) which is briefly described. The process of selecting comparison stars and the technique of detecting low-amplitude variables is discussed. The software is well suited to be used for on-line reductions at the telescope and a start has been made in this direction. Finally, some astrophysical results involving precision differential CCD photometry are described.
\end{abstract}

\section{INTRODUCTION}

Atmospheric extinction and scintillation is one of the largest sources of error in photometric observations. If one is interested in absolute photometry, one needs the best atmospheric conditions to obtain accurate results. However, the dependency on the atmosphere is greatly reduced for differential photometry, particularly with CCD's. A typical extinction coefficient in the $\mathrm{B}$ or $\mathrm{V}$ band is 0.2 . For two stars separated by three arc min (the size of a typical CCD frame), the difference in extinction is only $0.6 \mathrm{mmag}$ at an airmass $\sec \mathrm{z}=2$ which is comparable to the scintillation noise for a $1.0-\mathrm{m}$ telescope, but is typically much smaller than photon noise. It is clear that differential CCD photometry is capable of high precision provided suitably bright comparison stars are visible on the same frame.

For the last few years my colleagues and I have been using an RCA 360 x 512 CCD attached to the 1.0-m telescope at Sutherland to obtain differential photometry of young clusters in the galaxy and the Magellanic Clouds. The program consists of two parts: (a) to obtain accurate uvby $\beta$ photometry of cluster members and (b) to search for microvariables in the clusters. The purpose of obtaining Strömgren photometry of young open clusters is to refine the absolute magnitudes of early-type stars. The survey for microvariables is important in defining the instability strip for $\beta$ Cep, $\lambda$ Eri and 53 Per stars. The field of a galactic open cluster is normally too large to be covered by a single CCD frame: a mosaic of overlapping frames is required. The field of young LMC and SMC clusters is covered by a single frame, but is very crowded. In this paper we describe our techniques for differential CCD photometry, illustrating it with some of our results.

\section{INSTRUMENTATION AND OBSERVING TECHNIQUE}

The CCD camera is attached to a 1.0-m reflector. The filter box is situated some distance 
above the CCD chip in the converging beam. Ideally, the temperature of the filters should be kept within narrow limits since the characteristics of the narrow band filters vary with temperature, but no provision is made for this. As a result, some systematic errors may be introduced for the $\beta$ index, but the effect should be negligible for the other Strömgren filters. The CCD chip itself is kept at a temperature of about $158^{\circ} \mathrm{K}$.

A CCD can be modeled statistically by two parameters: $\lambda$ - the number of electrons per $\mathrm{ADU}$ and $\sigma_{\mathrm{r}}$ - the readout noise in electrons. The pixel-to-pixel variance, $\sigma^{2}$, versus the mean ADU count, $z$, can be used to determine the two constants $\lambda$ and $\sigma_{\mathrm{r} .}$ A number of exposures on the darkening sky soon after sunset were obtained through the y filter. As expected, the relationship between $\sigma^{2}$ and $z$ is linear, but there is a discontinuity in slope for $z>17000$. This discontinuity remains unexplained. If we restrict the analysis to $z<17000$ ADU we obtain:

$$
\begin{aligned}
& \lambda=10.91 \pm 0.08 \text { electrons/ADU, } \\
& \sigma_{\mathrm{r}}=87.7 \pm 0.4 \text { electrons. }
\end{aligned}
$$

Flatfield calibrations are made by observing a cloudless twilight sky. Experiments show quite conclusively that the sensitivity variation across the CCD is independent of time. This means that it is permissible to combine several flatfield calibrations for a given filter to improve the signal-to-noise ratio. Generally, from five to ten calibrations per filter, each with a mean ADU count of 15000 , were combined.

The RCA chip has a problem with charge transfer at low light levels which results in a nonlinearity for counts less than about 50 ADU. It is therefore necessary to expose the CCD to a uniform source of illumination to bring the ADU count above this level prior to each exposure. This is accomplished by a momentary illumination of the CCD by four LED's. These "preflashing" exposures need to be calibrated at regular intervals as the illumination level is somewhat dependent on time.

The calibration of the electronic bias is made using an overscan strip on the CCD. We assume that the bias is constant along the readout direction but varies perpendicular to this direction. The variation is modeled from the overscan using a suitable running mean.

Before a frame is subjected to astrophysical analysis, it undergoes the following "cleaning" processes:

a) electronic bias is subtracted,

b) bad pixels are patched up,

c) the frame is divided by a normalized flatfield and trimmed.

The software for cleaning the frames was designed for processing very large numbers and runs automatically in batch mode on a UNIX workstation.

\section{REDUCTION SOFTWARE}

The search for short-period microvariables in open clusters demands continuous exposures over several hours and generates large numbers of frames (1000 per week is typical). Software 
which demands user interaction is not suitable for such a task. The process of identifying stars and performing profile fitting and aperture photometry should run automatically in batch mode. We found DoPhot particularly suitable for this purpose. This software was written by Mateo and Schechter and we are greatly indebted to them for allowing us to use it. A brief description of the software is given in Mateo and Schechter (1989); a more detailed account can be found in Schechter, Mateo and Saha (1993).

The program looks for objects above a certain threshold level. It models particular objects such as a star, double star, cosmic ray, galaxy, etc. For example, the model for a star might be an elliptical Gaussian. The model for a galaxy might also be an elliptical Gaussian, but one which is significantly bigger than a star. Having found the stars, it updates the relevant model parameters and subtracts the modeled stars from the field. The threshold is lowered and the process repeated. In each pass, stars found during previous passes are put back on the frame and improved parameters are re-calculated. Since neighboring fainter objects have now been subtracted, the shape parameters are expected to be better than on the previous pass. Throughout the above process, DoPhot constructs and updates a noise image which provides weights for each pixel used in the non-linear least squares fitting of the stellar profiles. In the final pass, aperture magnitudes are also calculated by replacing each star on the star-subtracted frame and then removing it again. The sky values are therefore uncontaminated by neighboring stars.

In our version of DoPhot, a star is modeled by the function:

$$
I(x, y)=a_{1}+a_{4} /\left(1+t+\left\{t^{2}\right\} / 2+\left\{t^{3 n}\right\} / 12\right)
$$

where $t=0.5\left(\left\{x^{2}\right\} / a_{5}+2 a_{6} x y+\left\{y^{2}\right\} / a_{7}\right)$. Here $x$ and $y$ are measured relative to the center of the stellar profile $\left(a_{2}, a_{3}\right)$. The parameters, $a_{5}, a_{6}$ and $a_{7}$, define the PSF. It is found that these parameters are independent of position on the chip. The best estimate of the PSF is obtained by weighting the values of the three parameters for each star according to appropriate statistical criteria and calculating a weighted mean. Initial starting values are automatically provided by fitting a simple Gaussian profile to the two or three brightest stars in the field.

In conventional aperture photometry, the aperture size is normally fixed for the duration of the night even when there is a change of seeing. A change in aperture size introduces a discontinuity in the zero point which is more difficult to model than the continuous change associated with seeing variations. This restriction no longer applies in CCD photometry. In our version of DoPhot, aperture magnitudes are determined by calculating the total count inside a square area of size $b_{1}$. The sky is calculated from the weighted mean of the count in the annulus formed by $b_{1}$ and an outer square of size $b_{2}$ The values of $b_{1}$ and $b_{2}$ vary with seeing according to the following algorithm:

$$
\begin{aligned}
& b_{1}=4.5\left(\left\{a_{5}\right\}\left\{a_{7}\right\}\right)^{0.25} \\
& b_{2}=4\left\{b_{1}\right\} / 3
\end{aligned}
$$

These values were obtained empirically by minimizing the error in a sequence of about a dozen frames of a moderately crowded field. The algorithm works well: typical rms values for all-sky photometry of E-region standards are three to five mmags even in conditions of variable seeing. 
Although aperture photometry gives good results for the brightest stars, we found that profile-fitting magnitudes are just as accurate for bright stars and far more accurate for the faintest stars. Generally speaking, the rms scatter for profile-fitting magnitudes is a factor of four to five times smaller for fainter stars. We use aperture photometry only for the purpose of standardization. For differential photometry we only use the magnitudes given by profile fitting.

\section{MATCHING STARS IN DIFFERENT FRAMES}

The output from DoPhot consists of a file listing the positions and relative fit and aperture magnitudes of all stars in the frame. After a typical observing run there will be a large number of frames of the same field, but DoPhot would have given different numbers to the same stars in different frames. The next step is to obtain a uniform numbering scheme. This is accomplished by selecting one frame as a master copy to which the other frames are matched.

The algorithm to match stars in different frames involves the use of similar triangles. All possible triangles formed by the twenty brightest stars in the fiducial frame are used to construct a graph of smallest angle versus largest angle. The same is done for the frame under study. A cross-correlation between the two graphs is sufficient to identify the stars irrespective of the relative orientation and scale of the two frames. If at least five stars are identified in this way, the relative shift and orientation of the frames can be calculated. The remaining stars are easily matched using a suitable distance criterion. For example, if the positions agree within one or two pixels it can be assumed that it is the same star. The algorithm is very robust and works in all but a very small number of peculiar cases.

\section{CHOICE OF COMPARISON STARS}

There are two criteria for the choice of comparison stars: they must be visible in practically all frames and they should be relatively bright. The number of comparison stars depends on the field. The initial selection consists usually of the ten brightest stars. The zero point of each frame is adjusted so that the mean magnitude for these stars is zero. The rms scatter for each star is examined and those with the largest scatter removed from the list. The process is repeated until a list of at least five or six good comparison stars is obtained.

As an illustration, we will take some recent work on the galactic open cluster NGC 6134. This cluster was examined for variables by Kjeldsen and Frandsen (1989) who detected three $\delta$ Scuti variables in one CCD frame. Subsequently, it was put on the STACC network project which is an attempt to join observers with small telescopes equipped with CCD cameras into teams that can produce light curves for variables (particularly $\delta$ Scuti stars). As part of this collaborative effort, S. Frandsen and M. Viskum (Aahrus University) observed the cluster with the Dutch 0.9-m telescope at La Silla and L. Balona and C. Koen (SAAO) with the 1.0-m telescope at Sutherland over a two-week period in May/June 1993. Observations were made through the V filter only; a brief description of the project is given in Frandsen et al. (1994). The field includes three $\delta$ Scuti stars. For this field, seven comparison stars were chosen, details of which are given in Table 1. Exposure times were $90-120 \mathrm{~s}$ for each frame. 


\section{TABLE 1}

Comparison Stars for a Field in NGC 6134

Results from SAAO Observations

\begin{tabular}{cllllll}
\hline \hline Star & $\sigma$ & $\mathrm{N}$ & $\mathrm{V}$ & $\alpha$ & $\sigma \alpha$ & $\sigma_{\mathrm{c}}$ \\
\hline 141 & 2.5 & 1236 & 11.87 & -150 & 009 & 2.3 \\
129 & 2.2 & 1240 & 12.54 & -130 & 008 & 2.0 \\
162 & 2.6 & 1240 & 12.65 & -162 & 009 & 2.3 \\
46 & 3.4 & 1240 & 12.94 & -100 & 013 & 3.3 \\
87 & 3.5 & 1239 & 13.19 & -11 & 014 & 3.5 \\
67 & 3.8 & 1240 & 13.98 & -257 & 149 & 3.8 \\
\hline
\end{tabular}

$\sigma$ is the standard error of one observation in mmag;

$\mathrm{N}$ is the number of observations; $\mathrm{V}$ is the magnitude; $\alpha$ is the correlation with seeing and $\sigma \alpha$ its standard error; $\sigma_{c}$ is the same as $\sigma$ after decorrelation.

As shown in Table 1, the rms scatter for the comparison stars is only two to four mmag on the average. However, this is contaminated by one night when observations were made through a thin cloud layer. On this night the rms error is close to six mmag. If this night is removed, the mean rms scatter is about $2.5 \mathrm{mmag}$. This indicates that for exposures as short as two min, the variation of thin cloud cover across the $C C D$ field can significantly decrease the photometric accuracy.

\section{SEARCH FOR LOW AMPLITUDE VARIABLES}

Once the frames have been standardized in the manner described above, the next step is to search for variability among the stars. An obvious way to do this is to select those stars with the largest rms error taking the magnitude into account. While this works well for high-amplitude variables, it is not a satisfactory way to detect microvariables. The periodic variations of a star with an amplitude of a few mmags is completely swamped by photon noise and will not show any significant increase in rms scatter.

A far better method, and the one used for these projects, is to calculate and display the periodogram of each star. In our software package, twenty periodograms are computed simultaneously and displayed on the screen. Since the time of observation is the same for all stars in the field, the cpu time used for calculating twenty periodograms is not much larger than used for a single periodogram. Visual inspection of the periodograms is used to pick out stars which may be variable. Not all the stars that appear to have significant peaks in the periodogram are variable. Spurious peaks can be a result of contamination by a close companion, if the star is close to the edge of the chip, and many other reasons. Candidate stars selected in this way are examined in more detail to produce a list of certain or probable short-period variables. 


\section{DECORRELATION}

When there are two stars very close to each other, the stars will be treated independently on frames of good seeing, but could be treated as a single star on frames of poor seeing. One way of avoiding this problem is to re-run DoPhot with fixed coordinates. While this can be done, generally speaking the results of the multi-fit solution are not as good, so we have simply omitted these frames for those particular stars. Quite often this situation gives rise to spurious peaks in the peridogram and the star is mistakenly put on the list of possible variables. To avoid this problem we have adopted the technique of testing each variable for a possible correlation between magnitude and seeing. If such a correlation exists, the frames are examined for a close companion. If one is found, nights of poor seeing or omitted: often the star is discarded entirely.

This is a simple illustration of a more general procedure - that of decorrelation. The concept behind decorrelation is that the magnitude should not depend on parameters peculiar to the CCD or the atmosphere. If a correlation is found, it illuminates a potential problem and one must consider a corrective remedy as illustrated above. The question may be asked as to whether the observed scatter is consistent with that expected from photon noise and the properties of the CCD or whether there is a non-random component which could be calibrated and used to reduce the noise level. One may think of, for example, a change of sensitivity across the CCD due to incomplete flatfield calibration. In this case one might expect a correlation between the calculated magnitude and the position of the star on the CCD chip. A plot of magnitude versus $\mathrm{X}$ or $\mathrm{Y}$ position will then show a correlation. This is a purely hypothetical example which is probably not likely to occur, but it is useful to examine possible correlations between magnitude and other parameters for the comparison stars and the $\delta$ Scuti variables in the field of NGC 6134.

We examined possible correlations between the calculated magnitude and the following quantities: seeing, sky level, $\mathrm{X}$ position and $\mathrm{Y}$ position. Analysis of the comparison stars and $\delta$ Scuti variables showed that when a correlation exists, it is mostly with the seeing. Very little, if any, of the variation correlates with the other parameters. However, the correlation varies in strength and sign from star to star as shown in Table 1. The last column of this table shows the quantity $\alpha$ in the linear relationship $\mathrm{V}=\alpha \mathrm{x}+\beta$, where $\mathrm{x}$ is the seeing in arbitrary units. The three brightest stars show a highly significant correlation with seeing, but the sign of the correlation changes from star to star. The correlation has, however, only a minor effect in reducing the overall scatter because the seeing was good for the most of the run.

As another example, we show in Fig. 1 part of the light curve of one of the $\delta$ Scuti stars in NGC 6134 using the Sutherland and La Silla data. The bottom curve in the figure is the raw data. The standard deviation of the residuals from a sinusoid fit with $P=0.1324 \mathrm{~d}$ is $6.3 \mathrm{mmag}$. When these residuals are decorrelated for seeing, sky level and position, the scatter is reduced to only 3.7 mmag (top curve). Most of the effect comes from the La Silla data. This shows that decorrelation can offer a significant improvement in the signal-to-noise ratio, but it should be used with caution as there is a possibility of introducing spurious signals. 


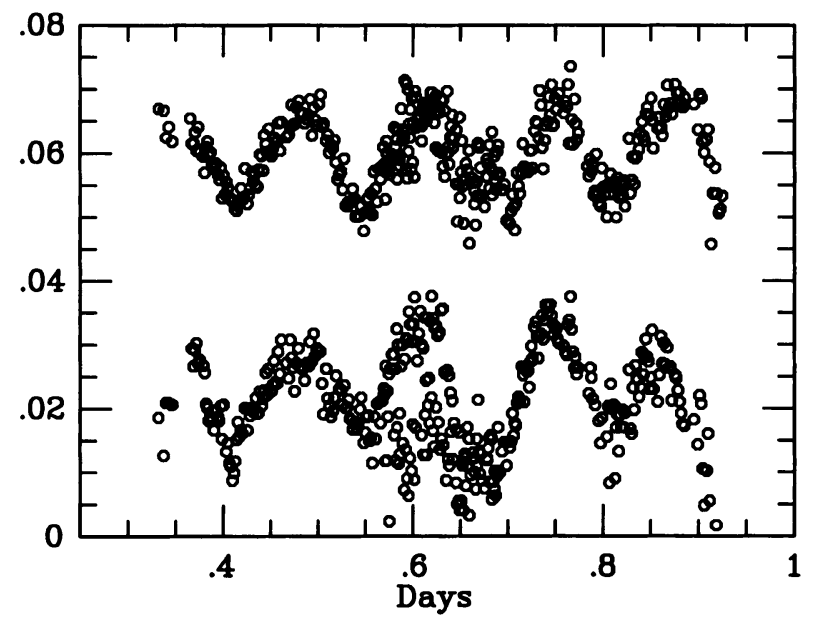

Fig. 1. The relative magnitude variation of a $\delta$ Scuti star in NGC 6134 (star 161) during one night from SAAO and ESO observations. Bottom curve - raw data; top curve - decorrelated data.

\section{THE NEED FOR ON-LINE REDUCTIONS}

Because DoPhot can run without any user intervention, it is well suited to be used for on-line reductions of stellar fields at the telescope. The usefulness of on-line reductions can only be appreciated once one has been exposed to it. For many years we have had on-line reductions on our photoelectric photometers which has resulted in greatly improved observing efficiency. For example, one can tell at a glance the quality if the observations and change observing plans as required. Stellar identification is much easier if one is presented with the magnitude and colors of the star just observed.

There is no doubt that implementation of on-line reductions for the CCD is a more complex problem. It needs to be tackled in several stages. The most important requirement is to provide an indication of the photometric quality of the night using all-sky aperture photometry. This is a relatively simple problem as it involves aperture photometry of the brightest star in the field. The next stage could involve obtaining profile-fitting photometry for a number of stars in the same field. It would not be practicable or necessary to provide the observer with a large list of all the stars identified by DoPhot - only marked stars or perhaps the ten brightest stars need be displayed. With this restriction the reduction time should be only a few seconds using a modern workstation, even in the most crowded fields. The final stage involves the running of DoPhot in the background to provide fully reduced data which may be inspected at any time during the night.

In all this, one needs adequate flatfield calibrations. These would not necessarily be available prior to observing, but for the first two stages an approximate flatfield calibration should provide the required information with sufficient accuracy. Such a project is being 
implemented at the SAAO by Dr. D. O'Donoguhe of the University of Cape Town.

\section{SOME ASTROPHYSICAL RESULTS}

In Fig. 2 we show the periodograms of four $\delta$ Scuti variables in NGC 6134 from the work of Frandsen, Viskum, Balona and Koen described above. These results are from a two-week run involving $1.0-\mathrm{m}$ class telescopes in Chile and South Africa and generated over 2000 frames of the same field in the cluster. These periodograms were calculated using the raw data; somewhat lower noise is obtained using decorrelation. The high-frequency noise level is about $0.5 \mathrm{mmag}$, allowing detection of periodicities with amplitudes in excess of one mmag for these 12 - 13 mag stars during bright time.

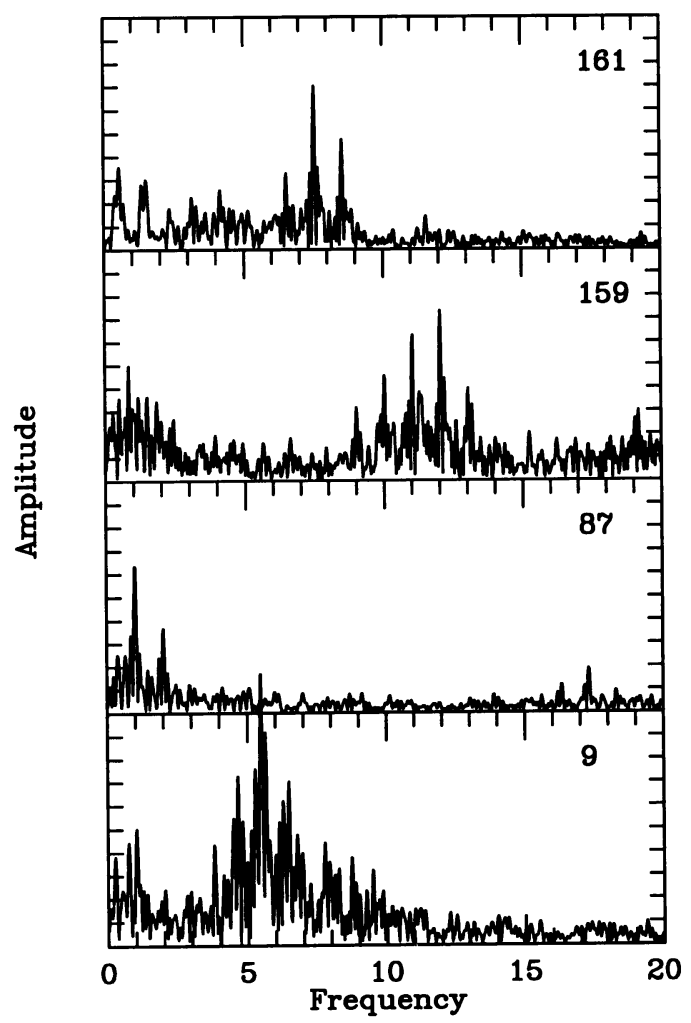

Fig. 2. Periodograms of four $\delta$ Scuti stars in NGC 6134 from SAAO and ESO observations. The frequency scale is in cycles/d; the tick marks on the amplitude axis are spaced by one mmag.

A breakthrough in our understanding of pulsation in early-type stars occurred in 1990 when it was realized that the metal opacities in use till then required a significant increase. This provided the driving mechanism for $\beta$ Cep variables which until this time had been a puzzle. The prediction is that early B-stars will pulsate provided the metal abundance is sufficiently high. To test this idea, Balona $(1992,1993)$ observed the cluster NGC 330 in the SMC and the 
clusters NGC 2004 and NGC 2100 in the LMC in the hope of detecting $\beta$ Cep stars. Even with a 1.0-m telescope and the high readout noise of the RCA CCD, it was possible to detect amplitudes as small as 0.01 for $16^{\text {th }}$ magnitude stars using a careful selection of comparison stars in these rather crowded fields. Although many variable stars, some with small amplitudes, were discovered, none of them satisfied the criteria defining the $\beta$ Cep class. Statistical analysis shows that the probability of this occurring by chance is less than 0.1 per cent, supporting the theoretical predictions.

A project of obtaining uvby $\beta$ photometry for young open clusters in collaboration with Drs. Koen and Laney (SAAO) is under way. Results from two clusters have already been published: NGC 3293 (Balona 1994) and NGC 4755 (Balona and Koen 1994). These clusters cover a large area of the sky: a mosaic of CCD frames with large overlap was used to transfer zero points from one frame to another. Within each frame comparison stars were used to obtain relative accurate photometry. Each cluster was also observed extensively to search for microvariables. This led to the discovery of one new $\beta$ Cep variable in NGC 3293 and six new $\beta$ Cep variables in NGC 4755 . No 53 Per variables were found, indicating that moderate and rapid rotation is likely to inhibit pulsations in these mid-B variables.

There is little doubt that the use of differential CCD photometry on small telescopes in bright time can make significant contributions to astrophysics, particularly stellar pulsation. Recent advances in computer technology and intelligent software has removed the main obstacle to efficient use of this technique - the reduction of large numbers of frames. In the near future it will be possible to obtain near-complete reductions at the telescope, further increasing the capabilities of the CCD.

\section{REFERENCES}

Balona, L. A. 1992 MNRAS 256, 425

Balona, L. A. 1993 MNRAS 260, 795

Balona, L. A. 1994 MNRAS 267. 1060

Balona, L. A. and Koen, C. 1994 MNRAS 267, 1071

Frandsen, S., Viskum, M., Balona, L. and Koen, C. 1994 Delta Scuti Star Newsletter 7, 5

Mateo, M. and Schechter, P. L. 1989 in 1st ESO/ST-EFC Data Analysis Workshop.

P. J. Grosbol, F. Murtagh and R. H. Warmels, eds, ESO, Garching, p. 69

Kjeldsen, H. and Frandsen, S. 1989 The Messenger 57, 48

Schechter, P. L., Mateo, M. and Saha, A. 1993 PASP 105, 1342

\section{DISCUSSION}

WALKER: For quick-look establishment of the zero-point while observing, you could just use aperture photometry and read the airmass from the header, to calculate a magnitude.

BALONA: Yes, but one would want this done automatically (i.e. without clicking on the star) at the end of the integration. When following a variable one would probably need the magnitudes of the comparison stars too.

ABBOTT: At which telescope have the ESO data been collected? 
BALONA: The 0.9-m Dutch telescope.

ABBOTT: Then that would explain why the decorrelation worked so well - the PSF is highly dependent on position in the image at that telescope.

BALONA: That may be the case.

PHILIP: I have used, some years ago, RCA chips at CTIO and KPNO. I found the $u$ magnitude to have a high rms error, even though the images on the frame looked fine. Do you have similar problems with your RCA chip? The newer TEK chips, however, give good u magnitudes.

BALONA: I have not found any problems peculiar to the $u$ filter.

HOWELL: I agree that while aperture photometry can be much worse than PSF fitting, that is generally only true for "standard" aperture photometry. Use of optimum aperture extraction techniques yields factors of two to three better than "standard", and is computationally faster than detailed PSF fitting.

BALONA: Yes. I was referring to measurements using a fixed aperture. 